\title{
Dichotomous Hypothesis of the Solar System Origin: Substantiation and Consequences for Astrobiology
}

\section{Vladimir Kompanichenko*}

Institute for Complex Analysis, FEB RAS 4, Birobidzhan, 679016, Russia

\begin{abstract}
The aim of this study consists in the advancement of the author's dichotomous hypothesis of hot origin of the solar system that is alternate to the well-known hypotheses of cold accretion. The hypothesis proposes formation of solar planets and satellites through dichotomous division of the superheated protoplanetary mass ejected from the youngest Sun due to strong non-equilibrium competition between gravitational contraction and heat expansion. Like the cold accretion hypotheses, the dichotomous hypothesis also explains the key regularities of the solar system structure (angular momentum distribution, position of the asteroid belt, back rotation of Venus and Uranus, etc.). Besides, it offers explanation of new data on extra solar planetary systems that is difficult to understand basing on the gradual cold accretion process (in particular, the misaligned protoplanetary discs in the binary protostar IRS 43). Some predictions following of the given explanation can be examined during future observations. The proposed hot origin of planets and satellites provides some new opportunities and directions for search of life in the solar system, including comets and hydrothermal environments on Mars and Europa.
\end{abstract}

Keywords: Solar system; Origin; Protoplanetary disc; Extra-solar planet; Satellite; Comet; Ejection; Life search

\section{Introduction}

There are different hypotheses devoted to explanation of the solar system origin. For the last some decades, the most of them are based on the idea that the solar planets formed within gas-dust cloud by means of gradual cold accretion. The scientists have elaborated various versions of the accretion process that are described in a lot of articles and books [1-3]. In framework of this approach, many basic facts of the solar system's structure have been satisfactory explained (for instance, angular momentum distribution, position of the asteroid belt, etc.). However, explanation of some other facts, like spatial distribution of the Jupiter's satellites or origination of the Kuiper belt, seems not so convincing. Besides, exploration of extra-solar planetary systems has brought unexpected discoveries, which contradict their emergence due to gradual cold accretion. For instance, recently three misaligned disks were found in the binary protostar IRS 43 [4]. The authors have come to the conclusion that turbulence has likely played a major role in the formation of IRS 43 (i.e., not a gradual accretion).

Taking the above into consideration, other little-known concepts of the origin of solar and extra-solar planetary systems should also be attracted for our attempts to explain new data obtained in astrophysics. One of such concepts is the dichotomous hypothesis [58]. It proposes the formation of solar planets and small bodies from the superheated protoplanetary mass ejected from the youngest Sun, with the following successive dichotomous (into two components) division. In the first half of $20^{\text {th }}$ century the idea about hot origin of solar system substantiated by J. Jeans was very popular. It implied an external cause of the hot protoplanetary mass ejection, just due to attraction of a passing star. By the $30-40^{\text {th }}$ years the mentioned external cause was evaluated as insufficient for ejection of the protoplanetary mass, with the following formation of hot solar planets; such evaluation gave rise to development of the cold accretion concept. However, in mid- $20^{\text {th }}$ century the opportunities of internal causes of the ejection were not investigated. Exploring them, the author formulated the dichotomous hypothesis focused on the internal cause of the superheat protoplanetary mass ejection. According to his supposition, the reason consists in the non-equilibrium competition between heat pressure and gravitational compression. It is assumed that the center of maximal temperature (the area with prevalent hydrogen) and the center of maximal gravitational attraction (the area with prevalent heavy elements) compete for location in the spatial center of a star. When the non-equilibrium degree exceeds a certain critical level, a star is able to undergo dichotomous division into two sub-equal or non-equal components. The last case corresponds with the ejection of a superheat protoplanetary mass from a young star. For the last decades it has become evident that the both phenomena-the accretion and plasma ejection are wide-spread in the Universe [9]. The remarkable example of the ejection event demonstrating emanation from very young stars is given by Reipurth and Bertout [10].

The aim of this article is to advance the dichotomous hypothesis establishing its correspondence with some new data on extra solar planetary systems. It will be shown that the dichotomous hypothesis, like the cold accretion hypotheses, is also able to explain key regularities of the Solar system's structure (angular momentum distribution, position of the asteroid belt, spatial distribution of the Jupiter's satellites, back rotation of Venus and Uranus, etc.). It also offers explanation of some new facts that refer to protoplanets, for instance, the turbulent formation of the multiple Keplerian disks in the binary protostar IRS 43. Based on the dichotomous hypothesis, some predictions in this way have been made, which can be examined during further exploration of extra solar planetary systems. Besides, some astrobiological consequences of the dichotomous hypothesis have been described as well.

*Corresponding author: Vladimir Kompanichenko, Institute for Complex Analysis, FEB RAS 4, Sholom Aleyhem Street, Birobidzhan, 679016, Russia, Tel: +7-4262224013; E-mail: kompanv@yandex.ru

Received July 07, 2016; Accepted November 18, 2016; Published January 12 2017

Citation: Kompanichenko V (2017) Dichotomous Hypothesis of the Solar System Origin: Substantiation and Consequences for Astrobiology. Astrobiol Outreach 5: 155. doi: 10.4172/2332-2519.1000155

Copyright: (c) 2017 Kompanichenko V. This is an open-access article distributed under the terms of the Creative Commons Attribution License, which permits unrestricted use, distribution, and reproduction in any medium, provided the original author and source are credited. 


\section{Ejection of the Protoplanetary Mass from Early Sun and Formation of Solar System: Proposing Mechanism and Consequences}

\section{Ejection of the protoplanetary mass from the Sun and its division: The initial supposition}

About 4.5-5 billion years ago the Sun was a very young star. From the dichotomous hypothesis point of view, a characteristic feature of very young stars implies that heat expansion may dominate over gravitational contraction. This is supported by observations of wellknown young $\mathrm{T}$ Tauri stars. Violent convection processes operate in them that one can interpret as a reflection of tense competition between the centres of mass and maximum temperature. A significant amount of matter outflows from their surface that emphasises a high role of heat pressure.

Early in the development, the young Sun was mainly comprised of hydrogen as well as minor amounts of heavy elements-silicon, magnesium, calcium, iron, etc. As the light elements (primarily hydrogen) were burning away and the end products of reactions were accumulating, the star was turning more opaque, and its core with a major portion of heavy elements was growing. It is supposed that then the core of heavy elements, along with some portion of light elements, was ejected from the early Sun by means of light (heat) pressure. The mechanic work to perform division of two bodies in the outer space was done by heat and kinetic energy of the Sun. The subsequent cycle of breaking up governed the formation of all the bodies from the ejected mass in the Solar system.

The protoplanetary mass was ejected from the central part of the Sun, where temperature can be arbitrary assessed at least some million degrees. As a result of the ejection, the mass passed into a quite different medium-outer space, where the temperature was near the absolute zero. It triggered the following chain of catastrophic consequences: an extremely rapid cooling of the outer parts of the superheat protoplanetary system; arising of a sharp temperature gradient between its inner and outer parts; increase of the force generated by heat pressure; the division of the initial protoplanetary mass into two components (inner and outer) due to ejection of the heavy core from it (Figure 1). A high proportion of silicon, magnesium, calcium, iron, and other elements that were disseminated throughout the protoplanetary mass were ejected ("filtered out") as well. Later during fast cooling significant part of the ejected elements were synthesized into molecules-the both simple $\left(\mathrm{H}_{2} \mathrm{O}, \mathrm{CO}_{2}, \mathrm{NH}_{3}\right.$, etc. $)$ and more complex (silicates, organics). The ejection was a chaotic process characterizing with intensive transfer of matter and energy due to convection. The division of the initial protoplanetary mass into two components was accompanied by a chaotic ejection of abundant small-sized clots of matter (Figure 2). Most of these clots (protoasteroids, protocomets, and protometeorites) were captured by the gravity fields of the inner and outer components and fell on them.

Clots of light protocomets and protometeorites ejected from the Sun could be thrown off to its distant orbits forming the Kuiper belt. The process of their hardening had to proceed very fast forming the dense cocoon around the Sun and protoplanets. Later most of them fell into Sun, planets and satellites during the period of heavy bombardment.

The proposed reconstruction conforms to the following fundamental regularities in the Solar system structure:

1. The Solar system is characterised by the following mass and angular momentum distribution: more than $99 \%$ of the mass is within the Sun, while $98 \%$ of angular momentum in the orbital movement of planets (formed in a course of the subsequent break-ups of the protoplanetary mass). According to the dichotomous hypothesis, the energy of the Sun rotation transfers into the forward movement of the protoplanetary mass due to the repulsion between the mass and temperature centres. The protoplanetary mass gained acceleration required to overcome the gravitational pull of the Sun whereas its rotation slowed down.

2. Planets of the Solar system are divided into two groups: low density outer planets and high density inner ones. According to the proposed reconstruction, this is due to the existence of inner and outer protoplanetary masses of contrasting densities that later on were subjected to further break up. The inner protoplanetary mass represented the heavy core ejected from the initial protoplanetary mass, whose discrete fragments (inner planets) were of high density (Figure 2). The outer Solar system's planets are discrete fragments of the outer component which ejected the core and disseminated heavy elements (as small clots) and, hence, was originally depleted in them. Being of high kinetic energy and weaker pulled to the Sun due low density, it moved to much longer distance from the Sun. TransNeptunian (Kuiper) comet belt, consisting of lightweight bodies, is situated behind the orbit of Neptune.

3. The asteroid belt is between the orbits of Mars and Jupiter, i.e., between the outer and inner planets. In the context of the proposed reconstruction, the belt's asteroids are congealed clots of matter ejected during the breakup of the initial protoplanetary mass and preserved own stable orbits due to location in the zone of equal gravitational attraction between the outer protoplanetary components and the Sun (with the small gravitational input of the inner components) (Figure 3). The asteroids, comets and meteorites with unstable orbits fell on planets and satellites during the period of heavy bombardment (4.6-3.9 billion years ago) and formed plentiful craters on their surfaces.

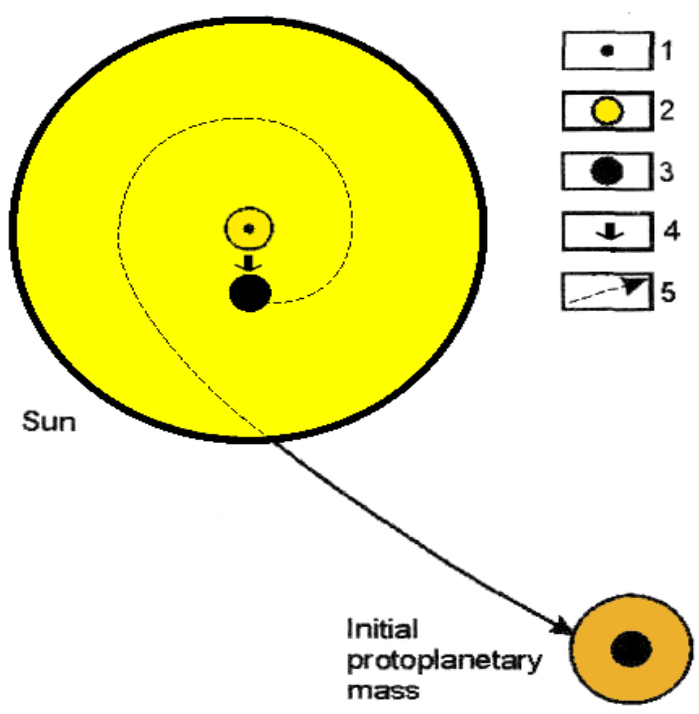

Figure 1: Ejection of the protoplanetary mass from the very young Sun about 5 billion years ago. 1) Geometric center of the Sun; 2) The maximum temperature center; 3 ) The core concentrating heavy elements; 4 ) Direction of repulsion of the core by heat pressure; 5) Schematic trajectory of the ejection. 


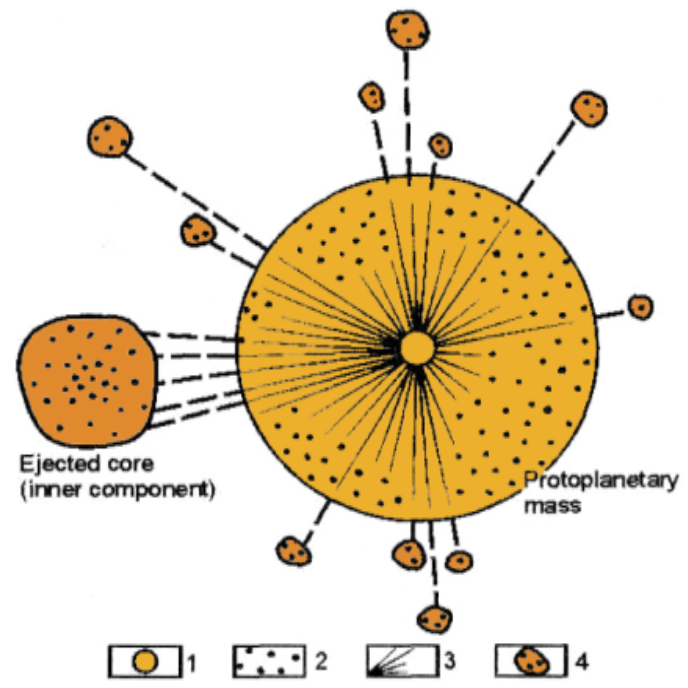

Figure 2: The first stage of the ejected protoplanetary mass division into the inner and the outer components: ejection of the heavy core (inner component) and smaller superheat clots. 1) Maximum temperature center; 2) Areas of high opacity in the protoplanetary mass (with high abundance of disseminated heavy elements); 3) Areas of intensive radiation (producing intensive radial and heat pressure); 4) Ejected superheat clots of matter containing significant amount of heavy elements: the core (inner component), protoasteroids, protocomets and protometeorites.

\section{Formation of the solar system planets}

The superheat inner and outer components were unstable systems. Being small in size, both components cannot maintain the same intensity of thermonuclear reactions in their interiors and irreversibly cool off. Using qualitative assessments as the base, both components though small in size, may be thought of as possessing a great store of free energy obtained from the Sun. Systems of this kind are capable of further division due to weak gravitational contraction force and high heat/radiation pressure. Excess free energy fuels this process.

A similar cycle of break up occurred as both components remain unstable and possess high energetic potential. The following reconstruction the most satisfactory explains the regularities of the Solar system structure. The inner component divided into two systemsproto-Mercury + proto-Venus and proto-Earth + proto-Mars, and the outer component into proto-Jupiter + proto-Saturn and proto-Uranus + proto-Neptune. In the subsequent cycle all the eight protoplanets became separated (Figures 4 and 5). Mars and Earth are very similar. The same concerns to the pairs Jupiter and Saturn, Uranus and Neptune. In addition, such reconstruction allows suggest explanation to the back rotation of Venus and Uranus. It can be supposed that these cycles of dichotomous division were accompanied by the ejection of smaller clots of matter (proto- asteroids, comets, meteorites).

This reconstruction is supported by the following facts.

1. The orbits of the eight planets are almost circular and lie in the equatorial plane of the Sun (maximum angle for Mercury is $7^{\circ}$ ). They move in their orbits in the same direction as the Sun. In the context of the proposed reconstruction the most probable spot for the ejection of the protoplanetary mass from the Sun is its equator. Parameters of planetary rotations locate the ejection spot in the equatorial plane of the Sun in the direction of its rotation.
2. The planetary rotation axes, as a rule, are not perpendicular to the ecliptic. It means that the planets formed through a complex chaotic process. This fact emphasizes an important role of the chaotic processes during division of the protoplanetary mass occurred due to surplus free energy in the primordial protoplanetary system.

3. Venus and Uranus rotate around their axes in the opposite direction compared with the other planets. As it was considered above, the breaking-up process was maintained by means of the repulsive forces. Action of these forces, in particular, explains angular momentum transfer from the Sun to the protoplanetary mass: the star slowed down its rotation whereas the ejected mass sped up that allowed it to reach the orbit around the Sun. A similar process occurred, when the protoplanets were separated. The inner protoplanetary mass was broken up in such a manner that the rotation of the protoEarth + proto-Mars system sped up (these planets rotate fast) while the rotation of the proto-Mercury + proto-Venus system slowed down (these planets rotate slowly). A repeat break up of proto-Mercury and proto-Venus triggered off rotation of the latter in the opposite direction. The similar process triggered off rotation of Uranus in the opposite direction in course of the proto-Uranus + proto-Neptune system division.

4. The present Earth and Venus are geologically active planets with intensive endogenous processes (volcanism, tectonics). The endogenous activity on Mars and Mercury is about ceased. The proposed reconstruction interprets these facts as follows. In the course of division the systems "proto-Earth + proto-Mars" and "proto-Mercury + proto-Venus" the protoplanets got different amounts of energy. The protoplanets with minor initial amount of heat energy (Mercury, Mars) cool off fast and the endogenous activity in them ceases. The protoplanets, which received a great surplus of heat energy, cool off slowly; their continuous geological evolution is sustained by the energy preserved in the interior.

\section{Formation of the planetary satellites}

The formation of planetary satellites is associated with further progressing of the 'chain reaction' of the protoplanetary mass disintegration. The separated hot protoplanets retain their ability to divide. Fast cooling of their surfaces and preserving of the superheat bowels led to extraordinary rise of the temperature gradient resulting

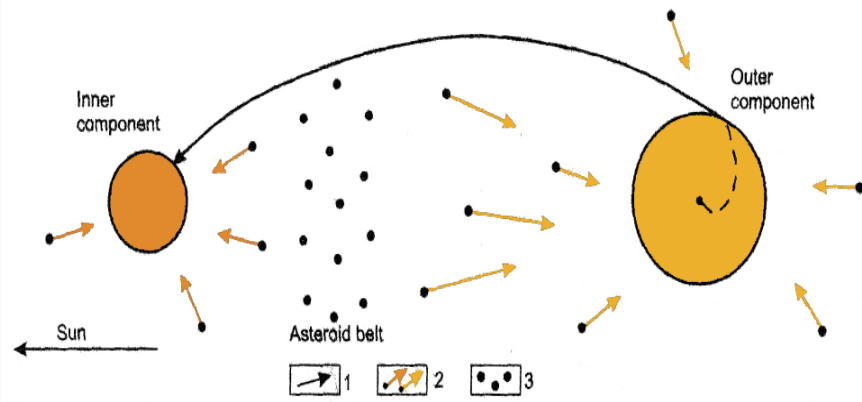

Figure 3: The scheme of directions of the asteroids movement after division of the initial protoplanetary mass. 1) Schematic trajectory of the inner component movement after division of the initial protoplanetary mass; 2) Protoasteroids possessing unstable orbits, directions of their fall; 3) Asteroids possessing stable orbits (inside the zone of equal attraction between the inner and the outer components), which form the asteroid belt. 


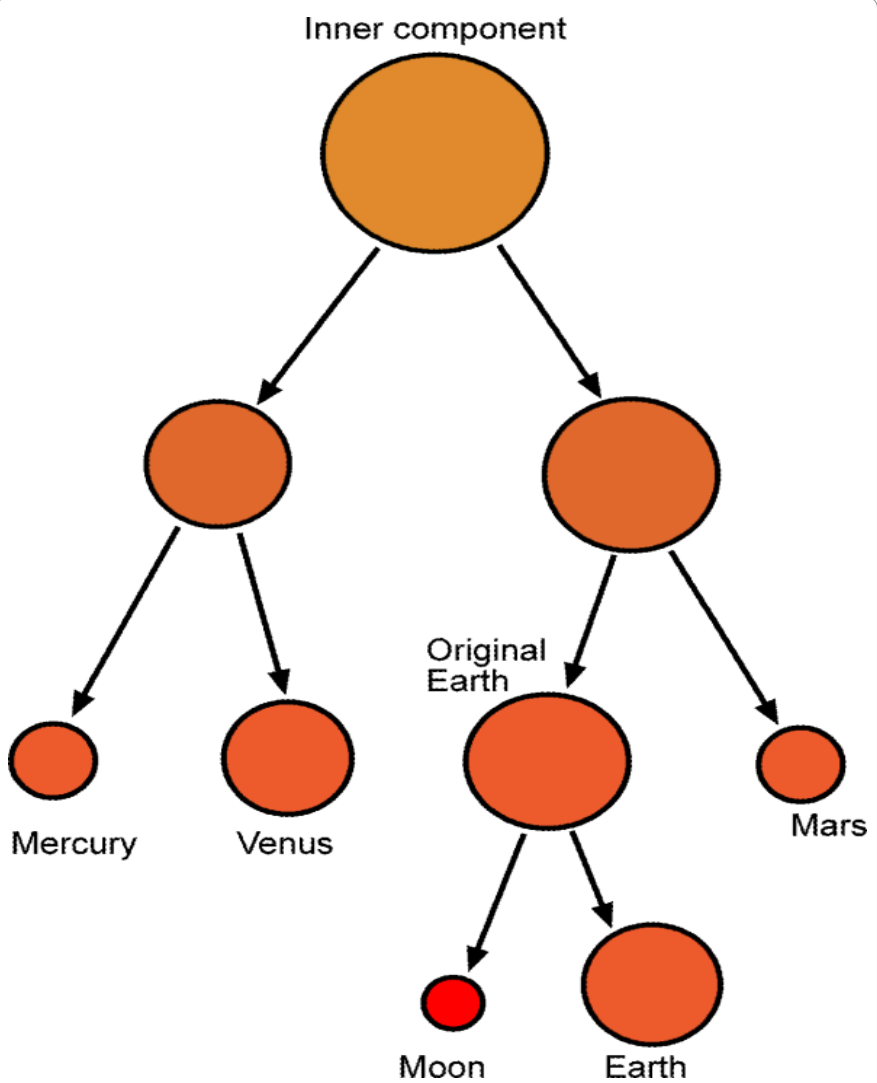

Figure 4: The cycles of dichotomous division of the inner component.

in intensive heat flow into outer space. According to the Onsager theorem, in a melt flow of heat inevitably initiates flow of components concentrations, and vice versa. Therefore, outflow of heat energy from the superheat protoplanets should be related with outflow of matter; and a catastrophic loss of heat energy at the initial stage of a protoplanets existence might generate a catastrophic ejection of matter. Following this supposition, protosatellites were ejected from the protoplanets in the manner described above. According to the made reconstruction, the given process developed in the inner and outer protoplanets in different paths.

In proto-Mercury, proto-Venus and proto-Mars (evidently, having no huge surplus of heat energy), the heavy core gradually occupied the protoplanet geometric centre. Apparently, the proto-Earth preserved enough surplus of heat energy to eject the proto-Moon. The outer protoplanets formed own satellite systems due to their large mass and significant store of heat energy. The heavy cores (as well as small clots of disseminated heavy elements) were ejected from their interiors. The ejected protosatellite masses underwent further fragmentation resulted in separation of hot protosatellites. As a rule, the kinetic energy was only enough for protosatellites to reach the orbit around the maternal protoplanets. In compliance with the proposed reconstruction, Pluto, being by origin a Neptune's satellite, received enough kinetic energy to overcome the gravitational attraction of Neptune and occupied the orbit around the Sun.

Let us consider, as an example, the formation of the Jupiter satellites' system. The physical parameters of the main satellites (with a mass of $0.2 \times 10^{16} \mathrm{~kg}$ and higher) are given in Table 1 .

As the table suggests, all the satellites can be classed into 3 groups of 4 objects each: the closest to Jupiter group (semi-major axis 128-
$222 \times 10^{3} \mathrm{~km}$, masses $0.2-208 \times 10^{16} \mathrm{~kg}$ ), the intermediate group (semimajor axis $422-1880 \times 10^{3} \mathrm{~km}$, masses $8932000-14820000 \times 10^{16} \mathrm{~kg}$ ), and the distant one (semi-major axis $11190-11780 \times 10^{3} \mathrm{~km}$, masses $0.6-670 \times 10^{16} \mathrm{~kg}$ ). In general, the satellites in each group have more or less comparable dimensions and rotate in closely spaced orbits. Orbits of some pairs of satellites are very close (Lysithea and Elara); besides, satellites Adrastea and Metis have coplanar orbits. Distances between the groups are significantly greater than those between the satellites within groups. In the context of the proposing hypothesis, the formation of the each group can be easily explained through double cycle of dichotomous division of a single superheat protosatellite. Other Jupiter's satellites are much smaller and rotate (at the exceptions of Themisto) in more distant orbits (primarily between $21000 \times 10^{3}$ $\mathrm{km}$ and $23500 \times 10^{3} \mathrm{~km}$ ). Their formation through possible multiple divisions demand special discussion.

So, the Solar system satellites were formed in course of the protoplanets division. The following facts support this reconstruction.

\begin{tabular}{|c|c|c|c|}
\hline Satellite & Semi-major axis $\left(\times 10^{3} \mathrm{~km}\right)$ & Diameter, km & Mass $\left(\times 10^{16} \mathrm{~kg}\right)$ \\
\hline Metis & 128 & $60 \times 40 \times 34$ & 3.6 \\
\hline Adrastea & 129 & $20 \times 16 \times 14$ & 0.2 \\
\hline Amalthea & 181 & $250 \times 146 \times 128$ & 208 \\
\hline Thebe & 222 & $116 \times 98 \times 84$ & 43 \\
\hline lo & 422 & 3660 & 8932000 \\
\hline Europa & 671 & 3120 & 4800000 \\
\hline Ganymede & 1070 & 5260 & 14820000 \\
\hline Callisto & 1880 & 4820 & 10760000 \\
\hline Leda & 11190 & 16 & 0.6 \\
\hline Himalia & 11450 & 170 & 670 \\
\hline Lysithea & 11740 & 36 & 6.3 \\
\hline Elara & 11780 & 86 & 87 \\
\hline
\end{tabular}

Table 1: Characteristics of the main Jupiter's satellites.

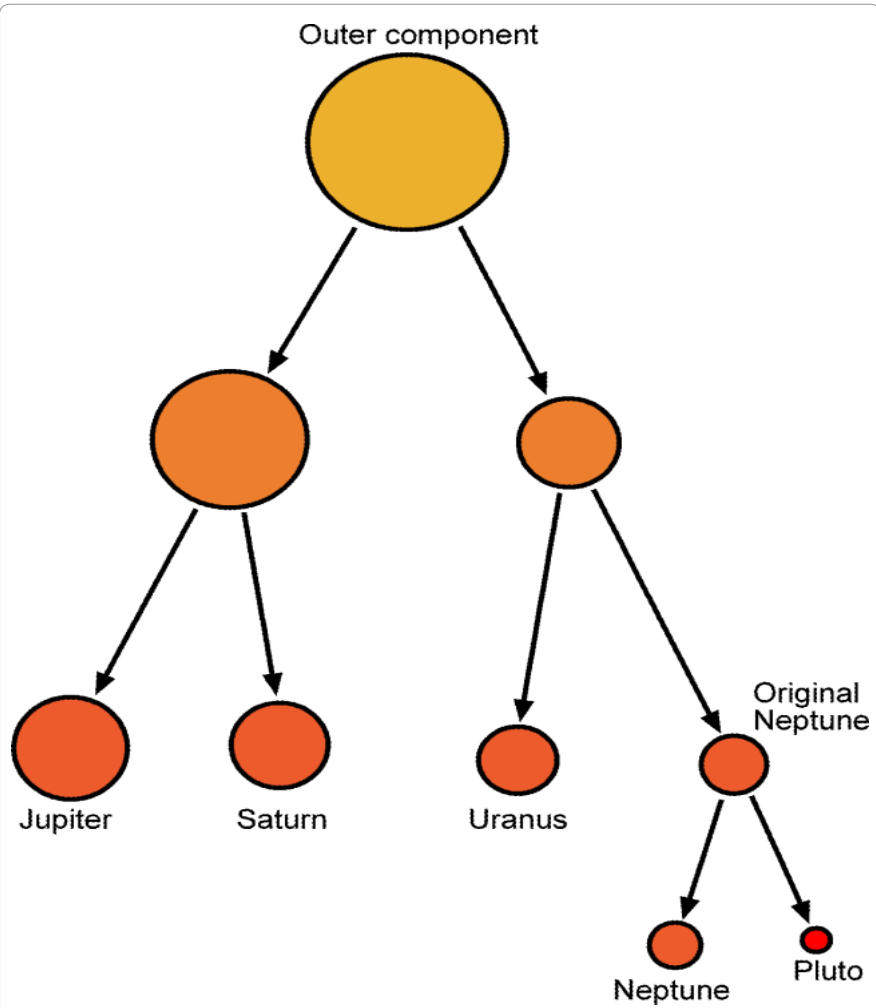

Figure 5: The cycles of dichotomous division of the outer component. 
1. Satellites of giant planets heavier than the maternal planets; in this context they similar to terrestrial planets. This fact is explained by the ejection of heavy cores from the light protoplanets during the formation of protosatellite systems.

2. The disposal the Galilean satellites around Jupiter are similar to the disposal of planets around the Sun: the closer to Sun/ Jupiter, the higher density of a body. This is related with the breakup of the ejected protosatellites mass in the gravitational field of Jupiter.

3. Among the Galilean satellites of Jupiter, Io and Callisto can be considered as 'antipodes'. Io is a geologically active body with active volcanism. There are no impact craters on its surface. The surface of Callisto is flickered by plentiful craters, and evidences of tectonic activity are absent there. According the proposed reconstruction, Io can be considered as the body which received a maximum of energy during the formation of the Galilean satellites. It has not cooled off yet and traces of the impact events have been eroded due to continuous geologic processes. Callisto received a minimum of energy. Its surface solidified fast and impact craters preserved on the surface.

4. Pluto differs from the planets by its elliptical orbit and its larger inclination to the ecliptic plane $\left(17,2^{\circ}\right)$. This fact, as well as the intersection of its orbit with the Neptune orbit, suggests that it was thrown out from the proto-Neptune and received a great bulk of kinetic energy in the course of ejection.

5. A fact that the Earth orbits the Sun is not precise. Actually, the mass centre of Earth-Moon system (that is located in the mantle of Earth in $4700 \mathrm{~km}$ from its geometric centre) orbits the Sun. This clarification implies that in the past the Earth and the Moon were a single body orbiting the Sun.

\section{Explanation of some regurarities in extrasolar planetary systems}

The best case to compare the cold accretion and the dichotomous hypotheses is the binary protostar IRS 43 explored by C. Brinch. The protostar is located in 400 light years from Earth. It consists of two new-born stars, around which revolve three protoplanetary discs that are significantly misaligned $\left(>60^{\circ}\right)$, both in inclination and position angle and also with respect to the binary orbital plane. Each stellar component has an associated circumstellar disk while the binary is surrounded by a circumbinary disk. The misalignment in this system suggests that turbulence has likely played a major role in the formation of IRS 43.

It seems difficult to offer reasonable explanation for the mentioned regularities in IRS 43 system basing on the gradual cold accretion concept. Using the dichotomous hypothesis, the following sketch of successive formation of the binary protostar IRS 43 can be preliminary outlined for discussion.

1. Dichotomous division of the initial protostar into two unequal components-the proper protostar I (bigger component) and the circumbinary disk (smaller component).

2. Dichotomous division of the protostar I into two sub-equal components-the protostar IA and the protostar IB.

3. Dichotomous division of the both protostars IA and IB into two unequal components: protostar IA and the circumstellar disk 1; protostar IB and the circumstellar disk 2.
As it was shown in the previous chapter, the ejections (or dichotomous divisions) of the superheat protoplanetary masses (further discs) were characterized by turbulent movement. This notion of the hypothesis can explain why the disks are significantly misaligned.

The given scheme can be examined in course of future IRS 43 exploration. It follows of the dichotomous hypothesis that dust of the discs represents itself hardened (micro) meteorites, which still have not been attracted by bigger bodies (planets, etc.). It is expected that the disks contain unseen hot planets, a part of which with satellites. Such supposition can be confirmed by indirect facts indicating possible unseen planets in other protoplanetary systems. Thus, availability of the unseen planet in the protoplanetary disk around TW Hya is supposed on basis of interpretation of the gap with a deficit of large grain.

\section{Astrobiological aspects of the dichotomous hypothesis}

According to the accepted definition, Astrobiology is an interdisciplinary science that studies the origin, evolution, and distribution of life in the Universe. A discovery of the first extrasolar planet in 1995 gave a great impulse to exploration of life in the Universe $[11,12]$. Various scientific approaches dealing with a search of life in space (Exobiology, Cosmo biology, Bio-astronomy, etc.) started integrating into one interdisciplinary science named Astrobiology. The main 10 goals of Astrobiology were formulated and published in the first issue of the journal Astrobiology [13]. Apparently, the study of life emergence and existence in the cosmic bodies of Solar System depends on the way they had formed (cold or hot).

There exist three accepted conditions for the origin of life: liquid medium, available organic compounds and energy source. One more necessary condition-significant thermodynamic and physicochemical fluctuations in the medium-was added by the author $[14,15]$. Taking into consideration the entire four conditions, hydrothermal systems represent the most probable medium for the origin of life. A lot of scientists support the hydrothermal scenario of life origin [1619]. From the point of view of the dichotomous hypothesis, volcanic and the associated hydrothermal processes are far consequences of the ejection event from the young Sun. This initial energy impulse gradually transformed and faded away in the following succession of events: ejection of protoplanetary mass $\rightarrow$ disintegration of the mass and the formation of proto-Earth $\rightarrow$ global liquid segregation and cooling of Earth $\rightarrow$ arise of volcanic and hydrothermal processes on the hardened surface $\rightarrow$ origin of life.

Taking into account some universal aspects of the dichotomous hypothesis, changeable hydrothermal media could serve as a potential cradle of life on various space bodies, including planets, satellites, and even smaller bodies [20]. Volcanoes and lava flows, as well as traces of hydrothermal activity, were found on some solar planets and satellites. For example, the diapirs in the ice crust of the Jupiter's satellite Europa represent the intrusions of warm ice in the circumjacent cold ice [21]. Such diapirs may appear at the expense of intensive heat flow rising from the liquid ocean, probably located beneath the ice cover. Another example is the discovered signs of relatively recently formed (10-100 million years ago or less) lava and water flows in Elysium and Amazonis Planitia regions of Mars, as it was reported by Sakimoto [22].

Volcanic and hydrothermal processes are impulsive, which is a result of the opposition of rising pressure of magma and fluid, on the one hand, and descending (lithostatic) pressure of the host rocks, on the other hand. An incessant interaction between these counter forces generates significant fluctuations of thermodynamic and physico- 
chemical parameters in hydrothermal systems that would allow the appearance of life. On the early Mars, 3-4 billion years ago, there existed the four conditions necessary for life emergence: the water ocean, organic compounds (recently discovered), volcanic activity-the energy source, and fluctuating hydrothermal medium. The same set of conditions could be on the satellite of Europa: a probable existence of the ocean (under the ice crust), displays of intensive tectonics in the crust indicative of submarine hot springs - the source of thermal/chemical energy and significant fluctuations. Although organic compounds have not yet been discovered on Europa, due to its remoteness from Earth, they are most likely to be there, because organic matter is wide-spread in the Universe. Of course, the availability of all these conditions on Mars and Europa does not mean an indispensable appearance and existence of life there.

It is an intriguing fact that comets can also provide the four necessary conditions for life emergence-only if they had formed in correspondence with the dichotomous hypothesis. Comets are composed of water ice and dust, and they often contain a lot of organic material. During the protoplanetary mass disintegration, powerful thermodynamic and physico-chemical fluctuations in superheated proto-comets could be maintained in them by intensive convection. But, though the initial forms of life could emerge in some comets, they could not reach the level of biological organization, comparable to that on Earth, because of their small size.

\section{Conclusion}

Unlike the well-elaborated cold accretion concept of the solar system origin, the dichotomous hypothesis represents a sketch that needs further elaboration. Nevertheless, the author have attempted to show that main regularities of the solar system structure can also be satisfactory explained basing on the idea of ejection of the superheat protoplanetary mass from young Sun. Moreover, the dichotomous approach offers the explanation of some unusual observations in extrasolar planetary systems (the binary protostar IRS 43) that is difficult to interpret following the cold accretion approach. The made predictions can be checked during future observations.

\section{References}

1. Vityazev AV, Pechernikova GV, Safronov VS (1990) Planets of the Earth's group: origin and early evolution. Her Russ Acad Sci 77: 232.

2. Boss AP, Durisen RH (2005) Chondrule-Forming Shock Fronts in the Solar Nebula: A Possible Unified Scenario for Planet and Chondrite Formation. Astroph J 621: 137

3. Greaves JS (2005) Disks around stars and the growth of planetary systems. Science 307: 68.

4. Brinch C, Jorgensen JK, Hogerheijde MR, Nelson RP, Gressel O (2016) Misaligned Disks in the Binary Protostar IRS 43. Astroph J Let 830: 16.

5. Kompanichenko VN (1993) Non-equilibrium of stars and the origin of the Solar system. DVNC RAEN, Khabarovsk.

6. Kompanichenko VN (2002) Non-equilibrium State of Stars and Dichotomous Formation of Planetary Systems, PGS Publisher, Khabarovsk.
7. Kompanichenko VN (2004) Dichotomous concept of planets origin: common pat of Mars and Earth. Proc. III European Workshop on Exo-Astrobiology, Madrid pp: 227-228.

8. Kompanichenko VN (2005) Thermal Energy and the Origin of Life. Front Perspect 14: 14-25.

9. Mirabel IF, Rodriguez LF (1999) A barred spiral at the centre of the giant elliptical radio galaxy Centaurus A. Astron Astroph 37: 409-443.

10. Reipurth B, Bertout (1997) IAU Symposium, no 182, Kluwer, Dordrecht.

11. Mayor M, Queloz D, Udry S, Halbwachs JL (1997) Astronomical and Biochemical Origins and the Search for life in the Universe. Editrice Cospositori pp: 313-330.

12. Marcy GW, Butler PR, Fisher DA, Vogt SS (2000) A New Era in Bioastronomy, ASP Conference Series 213: 85-94.

13. Morrison D (2001) NASA Astrobiology Programme. Astrobiol 1: 3-13.

14. Kompanichenko VN (2008) Three stages of origin of life process: Bifurication, Stabilization and inversion. Int J Astrobiol 7: 27-46.

15. Kompanichenko VN (2012) Inversion Concept of the Origin of Life. Orig. Life Evol Biosph 42: 153-178.

16. Corliss JB, Baross JA, Hoffman SE (1981) A hypothesis concerning the relationship between submarine hot springs and the origin of life on Earth Oceanol Acta Sp 4: 59-69.

17. Holm NG, Andersson E (2005) Hydrothermal simulation experiments as a too for studies of the origin of life on earth and other terrestrial planets: a review. Astrobiol 5: 444-460.

18. Russell MJ, Hall AJ, Boyce AJ, Fallick AE (2005) On Hydrothernal Convection Systems and the Emergence of Life. Econ Geol 100: 419-438.

19. Deamer DW (2011) First Life. University of California Press, Berkeley CA.

20. Kompanichenko VN (2009) Changeable hydrothermal media as potential cradle of life on a planet. Plan. Space Sci 57: 468-476.

21. Rathbun JG, Musser Jr, Squyres S (1998) Ice diapirs on Europa: Implications for liquid water. Geophys Res Let 25: 4157-4160.

22. Sakimoto S (2001) Volcanoes still Active on Mars? New Evidence for ongoing Volcanism and Water Release. Abstracts of the Geological Society of America Annual Meeting, Boston. 